\title{
FINITE GROUPS WITH FEW VANISHING ELEMENTS
}

\author{
Jinshan Zhang, Zhencai Shen and Jiangtao Shi \\ Sichuan University of Science and Engineering, China Agricultural \\ University and Yantai University, P. R. China
}

\begin{abstract}
Let $G$ be a finite group, and $\operatorname{Irr}(G)$ the set of irreducible complex characters of $G$. We say that an element $g \in G$ is a vanishing element of $G$ if there exists $\chi$ in $\operatorname{Irr}(G)$ such that $\chi(g)=0$. Let $\operatorname{Van}(G)$ denote the set of vanishing elements of $G$, that is, $\operatorname{Van}(G)=\{g \in G \mid \chi(g)=$ 0 for some $\chi \in \operatorname{Irr}(G)\}$. In this paper, we investigate the finite groups $G$ with the following property: $\operatorname{Van}(G)$ contains at most four conjugacy classes of $G$.
\end{abstract}

\section{INTRODUCTION}

Let $G$ be a finite group and $v(\chi):=\{g \in G \mid \chi(g)=0\}$, where $\chi$ is an irreducible complex character of $G$. A classical theorem of Burnside asserts that $v(\chi)$ is non-empty for all $\chi \in \operatorname{Irr}_{1}(G)$, where $\operatorname{Irr}_{1}(G)$ denotes the set of non-linear irreducible complex characters of $G$. Our aim in this paper is to analyze a particular subset of $\left\{x^{G}: x \in G\right\}$, which we denote by $\operatorname{Van}(G)$ and which encodes information coming from the set $\operatorname{Irr}(G)$ of irreducible complex characters of $G$. We say that an element $g \in G$ is a vanishing element of $G$ if there exists $\chi$ in $\operatorname{Irr}(G)$ such that $\chi(g)=0$ and otherwise we call $x$ a non-vanishing element. Let $\operatorname{Van}(G)$ denote the set of vanishing elements of $G$, that is

$$
\operatorname{Van}(G)=\{g \in G \mid \chi(g)=0 \text { for some } \chi \in \operatorname{Irr}(G)\} \text {. }
$$

Clearly, $\operatorname{Van}(G)$ is a proper normal subset of $G$. We denote $k_{G}(N)$ the number of conjugacy classes of $G$ contained in $N$, where $N$ is a normal subset of $G$. In this paper, we investigate the finite groups $G$ with the following property: $\operatorname{Van}(G)$ contains at most four conjugacy classes of $G$. Generally, we define

2010 Mathematics Subject Classification. 20C15.

Key words and phrases. Finite groups, characters, vanishing elements. 
Definition 1.1. A group $G$ is called a $V n C$-group if $\operatorname{Van}(G)$ contains at most $n$ conjugacy classes of $G$.

Clearly, if $G$ is a $V n C$-group, then, in particular every irreducible character of $G$ vanishes on at most $n$ conjugacy classes of $G$. However, it turns out (see [20] and Theorem 1.4 below) that for $n=3$ the two classes, in the case of solvable groups, in fact coincide. The aim of this paper is the classification of $V 4 C$-groups and the main results are the following.

TheOREM 1.2. Let $G$ be a finite non-abelian and solvable group. If $G$ is a V4C-group but not a V $3 C$-group, then one of the following is true:

(1) $G$ is a Frobenius group with complement $Q_{8}$,

(2) $G=G^{\prime} P$, where $G^{\prime}$ is a normal abelian 2-complement of $G, P \in$ $\operatorname{Syl}_{2}(G),|P|=8,|Z(G)|=4$, and $G / Z(G)$ is a Frobenius group with kernel $(G / Z(G))^{\prime} \cong G^{\prime}$ and complement $P / Z(G)$ of order 2 ,

(3) $G=\left(G^{\prime}\langle u\rangle\right) \times\langle t\rangle$, where $t$ is an involution and $G^{\prime}\langle u\rangle$ is a Frobenius group with kernel $G^{\prime}$ and complement of order 3 ,

(4) $G=G^{\prime} P$, where $G^{\prime}$ is a normal abelian 2-complement of $G$, and $P \in$ $\operatorname{Syl}_{2}(G),|P|=4, F(G)=G^{\prime}, G \backslash G^{\prime}$ is a union $x^{G} \cup y^{G} \cup z^{G} \cup h^{G}$ of four conjugacy classes satisfying $\left|C_{G}(x)\right|=4,\left|C_{G}(y)\right|=4,\left|C_{G}(z)\right|=6$ and $\left|C_{G}(h)\right|=12$,

(5) $G$ is a Frobenius group with abelian kernel $G^{\prime}$ and complement of order 5 .

THEOREM 1.3. Let $G$ be finite non-solvable group. If $G$ is a V4C-group, then $G$ is isomorphic to $A_{5}$.

Clearly, $\mathrm{A}_{5}$ is not a $V 3 C$-group. Hence by [20, Theorem A] and Theorem 1.3 above we easily get the following result.

TheOREM 1.4. Let $G$ be a finite non-abelian group. Then $G$ is a V3Cgroup if and only if $G$ is one of the following groups:

(1) $G$ is a Frobenius group with abelian kernel $G^{\prime}$ and complement of order 2

(2) $G$ is a Frobenius group with abelian kernel $G^{\prime}$ and complement of order 3

(3) $G \cong D_{8}$ or $Q_{8}$,

(4) $G$ is a Frobenius group with kernel $G^{\prime}$ and cyclic complement of order 4 ,

(5) $G=G^{\prime} P$, where $G^{\prime}$ is a normal and abelian 2-complement of $G, P \in$ $\operatorname{Syl}_{2}(G),|P|=4,|Z(G)|=2$, and $G / Z(G)$ is a Frobenius group with kernel $(G / Z(G))^{\prime} \cong G^{\prime}$ and complement $P / Z(G)$ of order 2 ,

(6) $G \cong S_{4}$

(7) $G=\left(G^{\prime}\langle t\rangle\right) \times\langle u\rangle$, where $\langle u\rangle$ is a cyclic group of order 3 and $G^{\prime}\langle t\rangle$ is a Frobenius group with kernel $G^{\prime}$ and complement of order 2 . 
In this paper, $G$ always denotes a finite group. Notation is standard and taken from [8]. In particular, denote $\pi_{e}(G)$ the set of all element orders of $G$, $\mathrm{C}(n)$ a cyclic group of order $n,(H, N)$ a Frobenius group with a complement $H$ and kernel $N$. we write $G=[F] H$ to denote a semidirect product of a normal subgroup $F$ and a subgroup $H$ of $G$. For $N \triangleleft G$, set $\operatorname{Irr}(G \mid N)=$ $\operatorname{Irr}(G) \backslash \operatorname{Irr}(G / N)$.

\section{NILPOTENT $V 4 C$-GROUPS}

In this paper, we shall freely use the following facts. Let $N \triangleleft G$ and write $\bar{G}=G / N$.

(1) For any $x \in G, \bar{x}^{\bar{G}}$ (when viewed as a subset of $G$, that is, the set $\left.\bigcup_{g \in G} x^{g} N\right)$ is a union of conjugacy classes of $G$. Note that $\left|C_{G}(x)\right|=$ $\left|C_{\bar{G}}(\bar{x})\right|+\sum\left\{|\chi(x)|^{2} \mid \chi \in \operatorname{Irr}(G \mid N)\right\}$ and that $k_{G}\left(\bar{x}^{\bar{G}}\right)=1$ if and only if $\left|C_{G}(x)\right|=\left|C_{\bar{G}}(\bar{x})\right|$, and then $k_{G}\left(\bar{x}^{\bar{G}}\right)=1$ if and only if $\chi(x)=0$ for all $\chi \in \operatorname{Irr}(G \mid N)$.

(2) If $G$ is a $V n C$-group, then so is $G / N$.

(3) Any character of $\bar{G}$ can be viewed, by inflation, as a character of $G$. In particular, if $x N \in \operatorname{Van}(\bar{G})$, then $x N \subseteq \operatorname{Van}(G)$.

(4) Let $M$ be a normal subgroup of $G$ contained in $N$. If $\psi \in \operatorname{Irr}(N)$ vanishes on $N \backslash M$, then by Clifford's theorem, every irreducible constituent of $\psi^{G}$ also vanishes on $N \backslash M$. In particular, $N \backslash M \subseteq$ $\operatorname{Van}(G)$.

We will use frequently the following lemma (see [16, Theorem 2.1]).

Lemma 2.1. Let $G$ be non-abelian, and let $\chi \in \operatorname{Irr}_{1}(G)$. Assume that $N$ is a normal subgroup of $G$ such that $G^{\prime} \leq N<G$. If $\chi_{N}$ is not irreducible, then the following two statements hold:

(1) There exists a normal subgroup $H$ of $G$ such that $N \leq H<G$ and $G-H \subseteq v(\chi)$.

(2) If $\left(G \backslash G^{\prime}\right) \cap v(\chi)$ consists of $n$ conjugacy classes of $G$, then $\left[H: G^{\prime}\right]([G$ : $H]-1) \leq n$.

The following result gives a lower bound on the number of conjugacy classes of zeros of irreducible characters of $p$-groups.

Lemma 2.2. [13, Theorem C] Let $\chi$ be a non-linear irreducible character of a p-group $P$ of degree $p^{n}$. Then $k_{G}(v(\chi))$ is a multiple of $p-1$ bigger than or equal to $(p+n)(p-1)$. In particular, $k_{G}(v(\chi)) \geq p^{2}-1$.

Next, we classify the non-abelian nilpotent groups in which every irreducible character vanishes on at most four conjugacy classes of $G$.

TheOrem 2.3. Suppose that $G$ is a non-abelian nilpotent group. If every irreducible character of $G$ vanishes on at most four conjugacy classes of $G$, then $G$ is one of the following groups: 
(1) $G \cong D_{8}$ or $Q_{8}$,

(2) $G \cong\left\langle a, b \mid a^{8}=b^{2}=1, b^{-1} a b=a^{-1}\right\rangle$,

(3) $G \cong\left\langle a, b \mid a^{8}=1, b^{2}=a^{4}, b^{-1} a b=a^{-1}\right\rangle$,

(4) $G \cong\left\langle a, b \mid a^{8}=b^{2}=1, b^{-1} a b=a^{3}\right\rangle$.

Proof. Take $\varphi \in \operatorname{Irr}_{1}(G)$ such that $\varphi_{G^{\prime}}$ is not irreducible. It follows from the hypothesis and Lemma 2.1 that $G$ has a proper subgroup $H$ such that $G^{\prime} \leq H<G, G-H \subseteq v(\varphi)$ and $\left[H: G^{\prime}\right]([G: H]-1) \leq 4$.

Since $G$ is nilpotent, it follows by Lemma 2.1 that $G$ is a 2 -group. Note that $\left[H: G^{\prime}\right]([G: H]-1) \leq 4$ and that $\left|G / G^{\prime}\right| \geq 2^{2}$; then we obtain that $\left|G / G^{\prime}\right|=4$ or 8 . Suppose that $\left|G / G^{\prime}\right|=8$. Let $N$ be normal in $G$ with $\left[G^{\prime}: N\right]=2$. Now consider the group $G / N$ of order 16 . Note that $\left[G / N:(G / N)^{\prime}\right]=8$, by the hypothesis, we easily conclude that it is impossible (see $\left[10\right.$, P. 300]). Hence we may suppose that $\left[G: G^{\prime}\right]=4$. If $\left|G^{\prime}\right|=2$, then $G$ satisfies (1) of the Theorem. So we may assume that $\left|G^{\prime}\right| \geq 4$. Now let $N$ be normal in $G$ with $\left[G^{\prime}: N\right]=4$. Consider the group $\bar{G}:=G / N$ of order 16 . Applying [10, P. 300], $G / N$ satisfies (2), (3) or (4) of the Theorem.

Now we show that $N=1$. Suppose that $N>1$. Set $M / N=Z(G / N)$. Take the irreducible character $\xi$ of $\bar{G}$ with $k_{\bar{G}}(v(\xi))=4$. Thus the hypothesis yields that $k_{\bar{G}}(v(\xi))=k_{G}(v(\xi))=4$, and so $\chi$ vanishes only on $v(\xi)$ for every $\chi \in \operatorname{Irr}(G \mid N)$. Hence it follows by $[9$, Theorem A] that $M=Z(G)$. Consequently, $[G: Z(G)]=8$.

Take an irreducible character $\rho$ of $\bar{G}$ with $k_{\bar{G}}(v(\rho))=3$. Choose $g \in G$ such that $\bar{g}=v(\rho) \backslash v(\xi)$. We easily see that $\bar{g}^{\frac{G}{G}}$ consists of two conjugacy class of $G$ and that $\bar{g}^{\bar{G}}=G^{\prime}-Z(G)$. Hence $k_{G}(G \backslash Z(G))=6$.

On the other hand, set $G \backslash Z(G)=n_{1}^{G}+\cdots+n_{s}^{G}$. Then, we get

$$
|G \backslash Z(G)|=\frac{|G|}{\left|C_{G}\left(n_{1}\right)\right|}+\cdots+\frac{|G|}{\left|C_{G}\left(n_{s}\right)\right|} .
$$

It follows that

$$
\frac{1}{\left|C_{G}\left(n_{1}\right)\right|}+\cdots+\frac{1}{\left|C_{G}\left(n_{s}\right)\right|}=1-\frac{1}{|G: Z(G)|}=\frac{7}{8} .
$$

Recall that all the centralizers of the elements in $G \backslash Z(G)$ have order greater than 4 , then $G \backslash Z(G)$ has at least 7 conjugacy classes, a contradiction. The proof is complete.

Remark 2.4. Clearly, a $V 4 C$-group is such a group whose irreducible characters vanish on at most four conjugacy classes. But the converse is not true (see the types (2), (3) or (4) in Theorem 2.3).

Corollary 2.5. Suppose that $G$ is a non-abelian nilpotent group. If $G$ is a $V 4 C$-group, then $G$ is isomorphic to $D_{8}$ or $Q_{8}$. 


\section{VANISHING ELEMENTS AND THE NILPOTENT NORMAL SUBGROUP}

In the following, we consider the situation in which a vanishing element of a group $G$ lies in a nilpotent normal subgroup.

LEMMA 3.1. Let $N$ be a nilpotent subgroup of $G$ such that $|G: N|=2$. Assume that $G$ is non-nilpotent. If $N$ is non-abelian, then $k_{G}(N \cap \operatorname{Van}(G)) \geq$ 3 .

Proof. Consider $\theta \in \operatorname{Irr}_{1}(N)$. Note that $|G: N|=2$, thus there exists $\chi \in \operatorname{Irr}(G)$ such that either $\chi_{N}=\theta$ or $\chi=\theta^{G}$. If $\chi_{N}=\theta$, then it follows by the nilpotency of $N$ and Lemma 2.1 that $k_{N}(v(\theta)) \geq 6$, and so $k_{G}(v(\theta)) \geq 3$. Thus $k_{G}(N \cap \operatorname{Van}(G)) \geq 3$. Hence we may assume that $\chi=\theta^{G}$.

Now let $M$ be a normal subgroup of $N$ maximal with respect to $N / M$ being non-abelian. Then $N / M$ is a $q$-group for some prime $q$ and $(N / M)^{\prime}$ is the unique minimal normal subgroup of $N / M$. Let $Z>M$ with $Z / M=$ $Z(N / M)$. Consider $\phi \in \operatorname{Irr}(N / M)$ with $\phi(1)=q^{n}>1$. The choice of $M$ implies that $\phi$ is a faithful irreducible character of $N / M$, and thus $\phi$ satisfies $\phi(1)^{2}=|N / M: Z / M|$ by [8, Theorem 2.31]. Applying [8, Corollary 2.30], $\phi$ vanishes on $N \backslash Z$.

From the first paragraph of the proof, we have that $\phi^{G}$ is an irreducible character of $G$. Take $\psi=\phi^{G}$. Observe that $\psi$ vanishes on $N \backslash\left(Z \cup Z^{x}\right)$, where $x \in G \backslash N$. Set $N \backslash\left(Z \cup Z^{x}\right)=n_{1}^{G}+\cdots+n_{s}^{G}$. Then, we get

$$
\left|N \backslash\left(Z \cup Z^{x}\right)\right|=\frac{|G|}{\left|C_{G}\left(n_{1}\right)\right|}+\cdots+\frac{|G|}{\left|C_{G}\left(n_{s}\right)\right|},
$$

and

$$
\left|N \backslash\left(Z \cup Z^{x}\right)\right|>|N|-2|Z|
$$

It follows that

$$
\frac{1}{\left|C_{G}\left(n_{1}\right)\right|}+\cdots+\frac{1}{\left|C_{G}\left(n_{s}\right)\right|}>\frac{1}{2}-\frac{1}{q^{2 n}} \text {. }
$$

Assume that $q=2$. Then 2||$N \mid$. Recall that $N$ is not a 2-group; thus $\left|C_{G}\left(n_{i}\right)\right| \geq 12$. Note that

$$
\frac{1}{\left|C_{G}\left(n_{1}\right)\right|}+\cdots+\frac{1}{\left|C_{G}\left(n_{s}\right)\right|}>\frac{1}{2}-\frac{1}{4}=\frac{1}{4} .
$$

Hence, $s \geq 4$. Now, we assume that $q$ is odd.

Since $q$ is odd, we get have $\left|C_{G}\left(n_{i}\right)\right| \geq 9$. On the other hand, we have

$$
\frac{1}{\left|C_{G}\left(n_{1}\right)\right|}+\cdots+\frac{1}{\left|C_{G}\left(n_{s}\right)\right|}>\frac{1}{2}-\frac{1}{9}=\frac{7}{18} \text {. }
$$

Therefore, $s \geq 4$ and we are done.

Arguing as in Lemma 3.1 we easily see that the following two results. 
LeMma 3.2. Let $N$ be a nilpotent normal subgroup of $G$ such that $\mid G$ : $N \mid=3$. Assume that $G$ is non-nilpotent. If every element of $N$ is a nonvanishing element of $G$, then $N$ is abelian.

Lemma 3.3. Assume that $G=N P$, where $N$ is a nilpotent normal 2complement of $G$ and $P \in \operatorname{Syl}_{2}(G)$. Assume that $|P|=4$. If every element of $N$ is a non-vanishing element of $G$, then $N$ is abelian.

Theorem 3.4. Let $G$ be Frobenius group with kernel $N$ and complement of order 3 . If $N$ is non-abelian, then $k_{G}(N \cap \operatorname{Van}(G)) \geq 3$.

Proof. Let $G$ be a minimal counter-example. Then $N$ is a $q$-group for some prime $q$, also $N^{\prime}$ is minimal normal in $G$ and $N^{\prime} \leq Z(N)$. Let $C=\langle x\rangle$ be a complement of $N$ in $G$.

Observe that any irreducible $C$-invariant subgroup of $N$ is of order at most $q^{3}$, also that if $q=2$ then the bound is $2^{2}$. In particular, $\left|N^{\prime}\right|=q^{e} \leq q^{3}$.

Let $\psi \in \operatorname{Irr}(N)$ be of maximal degree. Observe that there exists a subgroup $Z$ of $N$ such that $\psi$ vanishes on $N \backslash Z$ and that $Z \geq N^{\prime},|N / Z|=q^{2 m}$.

Suppose that $q>2$ or $m>1$. Then there exists an irreducible character $\chi$ of $G$ such that $\chi$ vanishes on $G \backslash \Delta$, where $\Delta:=Z \cup Z^{x} \cup Z^{x^{2}}$. Write $\left|Z / N^{\prime}\right|=q^{f}$. Observe that the centralizer of any element in $N \backslash N^{\prime}$ has order at least $\left|N / N^{\prime}\right|$. Now

$$
\begin{aligned}
k_{G}(G \backslash \Delta)-2 & =k_{G}(N \backslash \Delta) \\
& >\left(q^{2 m+f+e}-3 q^{f+e}\right) / 3 q^{e} \\
& =q^{f}\left(q^{2 m}-3\right) / 3>3,
\end{aligned}
$$

hence, we obtain a contradiction.

Suppose that $q=2$ and $m=1$. As $\psi$ is of maximal degree, $\operatorname{cd}(N)=$ $\{1,2\}$. It follows that either $|N: Z(N)|=8$ or $N$ has an abelian subgroup of index 2. Observe that if $N / Z(N)$ has order 8 , then $N / Z(N)$ has a $C$ invariant subgroup of order 2, which is impossible because $G$ is a Frobenius group. Assume now that $N$ has an abelian subgroup $E$ of index 2 and set $D=\cap_{g \in G} E^{g}$. Then all non-linear $\psi \in \operatorname{Irr}(N)$ vanishes on $N \backslash E$. Take $\chi \in \operatorname{Irr}(G)$ of degree 6 . We see that $\chi$ vanishes on $N \backslash E$, and it follows that $\chi$ vanishes on every element of $N \backslash D$. It follows by [9, Theorem A] that $Z(N) \leq D$. Then we get

$$
N^{\prime} \leq Z(N) \leq D \leq N .
$$

Observe that $|N / D| \geq 4$, thus we conclude

$$
4 \leq\left|N^{\prime}\right| \leq|Z(N)| \leq|D| \leq \frac{|N|}{4} .
$$

If all are equalities above, then $N$ is of order $16, N^{\prime}=Z(N)$ and $\left|N: N^{\prime}\right|=4$. However, it is impossible (see [10, P. 300]). Hence $|N| \geq 64$. Set $N \backslash D=$ 
$n_{1}^{G}+\cdots+n_{s}^{G}$. Thus, we have

It follows that

$$
|N \backslash D|=\frac{|G|}{\left|C_{G}\left(n_{1}\right)\right|}+\cdots+\frac{|G|}{\left.\mid C_{G}\left(n_{s}\right)\right) \mid} .
$$

$$
\frac{1}{\left|C_{G}\left(n_{1}\right)\right|}+\cdots+\frac{1}{\left|C_{G}\left(n_{s}\right)\right|}=\frac{1}{3}-\frac{1}{3|N: D|} \geq \frac{1}{4} .
$$

Observe that all the centralizers of the elements in $N \backslash D$ have order greater than 4, also that the centralizer of some element, say $g$, has order at least 32. So we conclude by the above equality $(*)$ that $N \backslash D$ consists of at least 3 conjugacy classes, a contradiction.

Arguing as in Theorem 3.4, we easily see that the following lemma.

Lemma 3.5. [1, Proposition 4.1] Let $G$ be Frobenius group with kernel $N$ and complement of order $p$, where $p \leq 5$. If $N \cap \operatorname{Van}(G)=\emptyset$, then $N$ is abelian.

\section{Conjugacy Classes outside a nORMal subgroup}

We will use frequently the following result.

Lemma 4.1. Let $G$ be non-abelian. Assume that $N$ is a normal subgroup of $G$ such that $G^{\prime} \leq N<G$ and that $G \backslash N \subseteq \operatorname{Van}(G)$. If $\operatorname{Van}(G)$ consists of at most $n$ conjugacy classes of $G$, then $\left|N: G^{\prime}\right|(|G: N|-1) \leq n$.

Proof. Assume that $\left|N: G^{\prime}\right|=m$ and $|G: N|=r$. Then we have

$$
G=N+N x_{1}+\ldots+N x_{r-1}, x_{i} \notin N,
$$

and

$$
N=G^{\prime}+G^{\prime} y_{1}+\ldots+G^{\prime} y_{m-1}, y_{j} \notin G^{\prime}
$$

It follows that

$$
G \backslash N=\sum_{i=1}^{r-1} \sum_{j=1}^{m-1} G^{\prime} y_{j} x_{i}+\sum_{i=1}^{r-1} G^{\prime} x_{i} .
$$

For $x \notin G^{\prime}, G^{\prime} x$ is a normal subset of $G$, and so we conclude by the above equality (*) that $G \backslash N$ consists of at least $m(r-1)$ conjugacy classes. Bearing in mind that $G \backslash N \subseteq \operatorname{Van}(G)$, by the hypothesis we obtain that $m(r-1) \leq n$, that is $\left|N: G^{\prime}\right|(|G: N|-1) \leq n$.

We will also make use of the following result, which is [9, Theorem 4.3]. As usual, we denote by $F(G)$ the Fitting subgroup of a group $G$.

LEMma 4.2. Let $G$ be a solvable group and let $x$ be an element of $G$ such that $\chi(x) \neq 0$ for every $\chi \in \operatorname{Irr}(G)$. Then the image of $x$ modulo $F(G)$ has 2-power order. 
Lemma 4.3. [4, Lemma 2.6] Let $G$ be a solvable group, and $N$ a normal subgroup of $G$. If $N / F(N)$ is abelian, then $N \backslash F(N) \subseteq \operatorname{Van}(G)$.

The following lemma is the key to the proof of Theorem 1.2.

LEMmA 4.4. Suppose that $G=K P$, where $K$ is a normal 2-complement of $G$ and $P \in \operatorname{Syl}_{2}(G)$. If $G$ is a $V 4 C$-group and $P$ is non-abelian, then one of the following statements holds:

(1) $K=1$ and $G \cong D_{8}$ or $Q_{8}$.

(2) $G$ is a Frobenius group with complement $Q_{8}$.

Proof. By Corollary 2.5, we may assume that $K>1$. Since $P$ is nonabelian, it follows by Corollary 2.5 that $G / K \cong P \cong \mathrm{Q}_{8}$ or $\mathrm{D}_{8}$. Hence we have that $\left|G / K: G^{\prime} K / K\right|=4$ and that $G \backslash G^{\prime} K \subseteq \operatorname{Van}(G / K)$. We then in a position to apply Lemma 4.1 to $G$, with $G^{\prime} K$ playing the role of $N$, obtaining that $K \leq G^{\prime}$ and so $G \backslash G^{\prime} \subseteq \operatorname{Van}(G)$. Observe that $k_{G}\left(G \backslash G^{\prime}\right)=3$ or 4 .

Assume that $k_{G}\left(G \backslash G^{\prime}\right)=3$. Set $G \backslash G^{\prime}=x G^{\prime}+y G^{\prime}+z G^{\prime}$. Thus, we get

$$
\left|G \backslash G^{\prime}\right|=\frac{G}{\left|C_{G}(x)\right|}+\frac{G}{\left|C_{G}(y)\right|}+\frac{G}{\left|C_{G}(z)\right|} .
$$

Then we conclude

$$
\left|C_{G}(x)\right|=\left|C_{G}(y)\right|=\left|C_{G}(z)\right|=4 \text {. }
$$

Hence every element in $P \backslash P^{\prime}$ acts fixed point freely on $K$. Therefore, since $P$ is a 2 -group of class 2 , we conclude by [11, Lemma 19.1] that $P \cong Q_{8}$ and $G$ is a Frobenius group with complement $Q_{8}$.

Assume that $k_{G}\left(G \backslash G^{\prime}\right)=4$. The hypothesis implies that $K \cap \operatorname{Van}(G)=$ $\emptyset$. Since $K$ is of odd order, it follows by Lemma 4.2 that $K \leq F(G)$. Consequently, $F(G)=K \times O_{2}(G)$. If $O_{2}(G)=1$, then, as $F\left(G^{\prime}\right)=F(G)=K$ and $\left|G^{\prime}: K\right|=2$, an application of Lemma 4.3 yields that $G^{\prime} \backslash K \subseteq \operatorname{Van}(G)$. Thus we obtain a contradiction. Therefore, $O_{2}(G)>1$.

Since $O_{2}(G)>1$, we get that $G^{\prime}$ is nilpotent, and also that $Z(G)=O_{2}(G)$. Set $G^{\prime}=Q$. We now show that $Q$ is abelian. Otherwise, Suppose that $Q$ is non-abelian. Consider $\theta \in \operatorname{Irr}_{1}(Q)$. Let $\chi \in \operatorname{Irr}\left(\theta^{G}\right)$. The hypothesis implies that $\chi_{Q}$ is not irreducible. Then by Clifford's theorem, $\chi_{Q}=e\left(\theta^{x_{1}}+\cdots+\theta^{x_{t}}\right)$, where $x_{1}, \cdots, x_{t}$ is a transversal of $\mathrm{I}_{G}(\theta)$ in $G$ and $e=\left[\chi_{Q}, \theta\right]$. Clearly, $t=2$ or 4. Let $M$ be a normal subgroup of $Q$ maximal with respect to $Q / M$ being non-abelian. Then $Q / M$ is a $q$-group for some prime $q$ and $(Q / M)^{\prime}$ is the unique minimal normal subgroup of $Q / M$. Let $Z>M$ with $Z / M=Z(Q / M)$. Consider $\theta \in \operatorname{Irr}(Q / M)$ with $\theta(1)=q^{n}>1$. Then, by [8, Corollary 2.30 and Theorem 2.31], $\theta$ vanishes on $Q \backslash Z$ and $|Q / Z|=q^{2 n}$. Notice that $\chi_{Q}$ vanishes on $Q \backslash\left(Z^{x_{1}} \cup \cdots \cup Z^{x_{t}}\right)$. The hypothesis yields that $Q=Z^{x_{1}} \cup \cdots \cup Z^{x_{t}}$ and thus $|Q| \leq 4|Z|$. Hence $|Q / Z| \leq 4$, thus we have reached a contradiction (note that $q$ is odd), and so $G^{\prime}$ is abelian. 
Set $G \backslash G^{\prime}=x G^{\prime}+y G^{\prime}+z G^{\prime}, x, y, z \in G \backslash G^{\prime}$. Since $k_{G}\left(G \backslash G^{\prime}\right)=4$, we may assume that both $x G^{\prime} \cup y G^{\prime}$ and $z G^{\prime}$ are a union of two conjugacy classes of $G$, respectively. Set $z G^{\prime}=z^{G}+h^{G}$. Recall now that $G / G^{\prime} \cong P / P^{\prime}$ is an elementary abelian group of order 4, there exists a normal subgroup $N$ of $G$ such that $G^{\prime}<N<G$, also that $|G: N|=2$ and $G \backslash N=x G^{\prime}+y G^{\prime}$. Thus we have

and

$$
\left|G \backslash G^{\prime}\right|=\frac{|G|}{\left|C_{G}(x)\right|}+\frac{|G|}{\left|C_{G}(y)\right|}+\frac{|G|}{\left|C_{G}(z)\right|}+\frac{|G|}{\left|C_{G}(h)\right|},
$$

It follows that

$$
\left|C_{G}(x)\right|=\left|C_{G}(y)\right|=4 .
$$

$$
\frac{3}{4}=\frac{1}{4}+\frac{1}{4}+\frac{1}{\left|C_{G}(z)\right|}+\frac{1}{\left|C_{G}(h)\right|} .
$$

Therefore, we easily see that $\left|C_{G}(z)\right|=\left|C_{G}(h)\right|=8$ or $\left|C_{G}(z)\right|=6$ and $\left|C_{G}(h)\right|=12$.

Suppose that $\left|C_{G}(z)\right|=6$ and $\left|C_{G}(h)\right|=12$. As $\left[G: G^{\prime}\right]=4$, the order of $z$ is even. Note that $|Z(G)|=2$, thus it is easy to see that 4 divides $\left|C_{G}(z)\right|$, a contradiction. Hence $\left|C_{G}(z)\right|=\left|C_{G}(h)\right|=8$, then every element in $P \backslash P^{\prime}$ acts fixed point freely on $K$. Observe that $G$ is a Frobenius group with kernel $K$ and complement $Q_{8}$, and thus $k_{G}\left(G \backslash G^{\prime}\right)=3$, a contradiction. The contradiction completes the proof.

The following result, which appears as [15, Theorem 2.2], will turn out to be useful in handling the case that $k_{G}(G \backslash N)=2$ and that $G \backslash N \subseteq \operatorname{Van}(G)$, where $N$ is normal in $G$.

Lemma 4.5. Let $N$ be a normal subgroup of a non-abelian solvable group $G$. Then $k_{G}(G \backslash N)=2$ if and only if $G$ is one of the following solvable groups.

(1) $N=1$ and $G \cong S_{3}$.

(2) $|G / N|=3$ and $G$ is a Frobenius group with kernel $N$.

(3) $|G / N|=2$ and $\left|C_{G}(x)\right|=4$ for all $x \in G \backslash N$. In particular, $P \in$ $\operatorname{Syl}_{2}(G)$ has a cyclic subgroup of order $|P| / 2$; furthermore, one of following holds:

(3.a) $G$ has a normal and abelian 2-complement.

(3.b) $G$ has a normal 2-complement and $P$ is a quaternion group.

(3.c) $G$ has an abelian 2-complement and $P \cong D_{8}$, the dihedral group of order 8 .

Proposition 4.6. Let $N$ be a normal subgroup of a non-abelian solvable group $G$. Assume that $k_{G}(G \backslash N)=2$ and that $G \backslash N \subseteq \operatorname{Van}(G)$. If $G$ is a $V 4 C$-group, then $G$ is one of the following solvable groups:

(1) $G$ is a Frobenius group with kernel $N$ and complement of order 3 ,

(2) $G \cong D_{8}$ or $Q_{8}$,

(3) $G$ is a Frobenius group with complement $Q_{8}$, 
(4) $G=G^{\prime} P$, where $G^{\prime}<N$ is a normal 2-complement of $G$, and $P \in$ $\mathrm{Syl}_{2}(G),|P|=4$,

(5) $G^{\prime}=N,\left|G: G^{\prime}\right|=2$ and $G / O_{2^{\prime}}(G) \cong S_{4}$.

Proof. By the hypothesis and Lemma 4.5, $G$ satisfies the condition of Lemma 4.1. Then we conclude by Lemma 4.1 that one of the following three cases occurs: (i) $\left|G: G^{\prime}\right|=3, G^{\prime}=N$; (ii) $\left|N: G^{\prime}\right|=2,|G: N|=2$; (iii) $G^{\prime}=N,\left|G: G^{\prime}\right|=2$.

Assume that $\left|G: G^{\prime}\right|=3, G^{\prime}=N$. Then by Lemma 4.5 , we have that $G$ is a Frobenius group with kernel $G^{\prime}$ and complement of order 3 .

Assume that $\left|N: G^{\prime}\right|=2,|G: N|=2$. Then it follows by Lemma 4.5 that $G=K P$, where $K \leq G^{\prime}$ is a normal 2-complement of $G$ and $P \in$ $\operatorname{Syl}_{2}(G)$. If $P$ is non-abelian, then by Lemma 4.4 we conclude that $G$ satisfies (2) or (3) of the Proposition. Hence we may assume that $P$ is abelian, then we easily see that $G=G^{\prime} P$, where $G^{\prime}<N$ is a normal 2-complement of $G$, and $P \in \operatorname{Syl}_{2}(G),|P|=4$.

Assume that $G^{\prime}=N,\left|G: G^{\prime}\right|=2$. Recall that $\left|C_{G}(g)\right|=4$ for any $g \in G \backslash G^{\prime}$. Take $y \in G$ with $T=C_{G}(y)$ of order 4. Clearly $T \subseteq C_{G}(T) \subseteq$ $C_{G}(y)=T$. Let $O_{2^{\prime}}(G)$ be the largest normal subgroup of odd order in $G$. Notice that $G$ is solvable, we use $[19$, Theorem 1,2] to conclude that $G / O_{2^{\prime}}(G) \cong \mathrm{S}_{4}$. The proof is complete.

The following proposition, which comes from [15, Theorem 3.6] and [17, Theorem 1.1], will turn out to be useful in handling the case that $G$ has a normal subgroup $N$ such that $k_{G}(G \backslash N)=3$ and that $G \backslash N \subseteq \operatorname{Van}(G)$, where $N$ is normal in $G$.

Proposition 4.7. Let $N$ be a normal subgroup of a non-abelian solvable group $G$. Then $G \backslash N$ is a union $x^{G} \cup y^{G} \cup z^{G}$ of three conjugacy classes satisfying $\left|x^{G}\right| \geq\left|y^{G}\right| \geq\left|z^{G}\right|$ if and only if one of the following is true:

(1) $N=1$ and $G \cong A_{4}$ or $D_{10}$,

(2) $G / N \cong S_{3}$ and $G \cong S_{4}$,

(3) $G$ is a Frobenius group with kernel $N$ and cyclic complement of order 4 ,

(4) $G \cong D_{8}$ or $Q_{8}$,

(5) $|G / N|=4$ and $G$ is a Frobenius group with complement $Q_{8}$,

(6) $|G / N|=2,\left|C_{G}(x)\right|=\left|C_{G}(y)\right|=\left|C_{G}(z)\right|=6$. In this case, $N$ is of odd order and $N$ has a normal and abelian 3-complement,

(7) $|G / N|=2,\left|C_{G}(x)\right|=4,\left|C_{G}(y)\right|=6$ and $\left|C_{G}(z)\right|=12$ and in this case, either $G$ has a normal 2-complement or $G / O_{2^{\prime}}(G) \cong S_{4}$,

(8) $|G / N|=2,\left|C_{G}(x)\right|=4,\left|C_{G}(y)\right|=\left|C_{G}(z)\right|=8$ and in this case, either $G / O_{2^{\prime}}(G) \cong G L(2,3)$ with abelian $O_{2^{\prime}}(G)$, or $G / O_{2^{\prime}}(G)$ is isomorphic to a non-abelian group of order 16 . 
Lemma 4.8 ([20, Lemma 2.7]). Let $G$ be a meta-abelian group. If $[G$ : $\left.G^{\prime}\right]=p$, then $G$ is a Frobenius group with kernel $G^{\prime}$ and complement of order $p$.

The following result will turn out to be useful in handling the case that $G / F(G)$ is abelian.

THEOREM 4.9. Let $G$ be a non-nilpotent group such that $G / F(G)$ is abelian. Assume that $k_{G}(G \backslash F(G))=4$. If $G$ is a V4C-group, then $G$ is one of the following groups:

(1) $G=G^{\prime} P$, where $G^{\prime}$ is a normal abelian 2-complement of $G, P \in$ $\operatorname{Syl}_{2}(G),|P|=8,|Z(G)|=4$, and $G / Z(G)$ is a Frobenius group with kernel $(G / Z(G))^{\prime} \cong G^{\prime}$ and complement $P / Z(G)$ of order 2 ,

(2) $G=\left(G^{\prime}\langle u\rangle\right) \times\langle t\rangle$, where $t$ is an involution and $G^{\prime}\langle u\rangle$ is a Frobenius group with kernel $G^{\prime}$ and complement of order 3 ,

(3) $G=G^{\prime} P$, where $G^{\prime}$ is a normal abelian 2-complement of $G$, and $P \in$ $\operatorname{Syl}_{2}(G),|P|=4, F(G)=G^{\prime}, G \backslash G^{\prime}$ is a union $x^{G} \cup y^{G} \cup z^{G} \cup h^{G}$ of four conjugacy classes satisfying $\left|C_{G}(x)\right|=4,\left|C_{G}(y)\right|=4,\left|C_{G}(z)\right|=6$ and $\left|C_{G}(h)\right|=12$,

(4) $G$ is a Frobenius group with abelian kernel $G^{\prime}$ and complement of order 5 .

Proof. Since $G / F(G)$ is abelian, it follows the hypothesis and by Lemma 4.3 that $G \backslash F(G)=\operatorname{Van}(G)$, and so $|G: F(G)| \leq 5$.

CASE 1. $|G: F(G)|=2$.

In this case, the hypothesis together with Lemma 3.1 yields that $F(G)$ is abelian. Notice that $G=K P$, where $K$ is a normal abelian 2-complement of $G$ and $P \in \operatorname{Syl}_{2}(G)$. Then, as $k_{G}(G \backslash F(G))=4$ and $G / F(G)$ is abelian, it follows by Lemma 4.4 that $P$ is abelian. Consequently, $G^{\prime} \leq K$. Applying Lemma 3.1, we conclude that $|P| \leq 8$ and that one of the following two cases occurs: (i) $\left|F(G): G^{\prime}\right|=1,2$ or 4 ; (ii) $\left|F(G): G^{\prime}\right|=3$.

CASE 1.1. $\left|F(G): G^{\prime}\right|=1,2$ or 4 .

First, assume that $F(G)=G^{\prime}$. Since $G^{\prime}$ is abelian, it follows by Lemma 4.8 that $G$ is a Frobenius group with abelian kernel $G^{\prime}$ and complement of order 2 , and so $k_{G}(G \backslash F(G))=1$, a contradiction.

Second, assume that $\left|F(G): G^{\prime}\right|=2$. Then $\left|G: G^{\prime}\right|=4$, and thus $G=G^{\prime} P$, where $G^{\prime}$ is a normal 2-complement of $G$ and $|P|=4$. Observe that $G / O_{2}(G)$ is a Frobenius group with Frobenius kernel $\left(G / O_{2}(G)\right)^{\prime} \cong G^{\prime}$ and complement of order 2. Thus $k_{G}(G \backslash F(G))=2$, a contradiction.

Finally, assume that $\left|F(G): G^{\prime}\right|=4$. Thus $\left|G: G^{\prime}\right|=8$ and so $G=G^{\prime} P$, where $G^{\prime}$ is a normal 2-complement of $G$ and $|P|=8$. Clearly, $\left|O_{2}(G)\right|=4$, and thus $\left|G / O_{2}(G):\left(G / O_{2}(G)\right)^{\prime}\right|=2$. Furthermore, applying again Lemma 4.8, we see that $G / O_{2}(G)$ is a Frobenius group with Frobenius kernel $\left(G / O_{2}(G)\right)^{\prime} \cong G^{\prime}$ and complement of order 2. Thus $G$ satisfies (1) of the theorem. 
CASE 1.2. $\left|F(G): G^{\prime}\right|=3$.

Recall now that $G^{\prime} \leq K \leq F(G)$, then $K=F(G)$ and so $|P|=2$. Observe that $G \backslash F(G)=x G^{\prime}+y G^{\prime}+z G^{\prime}$, where $x, y, z \in G \backslash F(G)$. Since $k_{G}(G \backslash F(G))=4$, we may assume that both $x G^{\prime} \cup y G^{\prime}$ and $z G^{\prime}$ are a union of two conjugacy classes of $G$, respectively. Set $z G^{\prime}=z^{G}+h^{G}$. we have

$$
|G \backslash N|=\frac{|G|}{\left|C_{G}(x)\right|}+\frac{|G|}{\left|C_{G}(y)\right|}+\frac{|G|}{\left|C_{G}(z)\right|}+\frac{|G|}{\left|C_{G}(h)\right|},
$$

and

It follows that

$$
\left|C_{G}(x)\right|=\left|C_{G}(y)\right|=6 .
$$

$$
\frac{1}{2}=\frac{1}{6}+\frac{1}{6}+\frac{1}{\left|C_{G}(z)\right|}+\frac{1}{\left|C_{G}(h)\right|} .
$$

Hence $\left|C_{G}(z)\right|=\left|C_{G}(h)\right|=12$, or $\left|C_{G}(z)\right|=8$ and $\left|C_{G}(h)\right|=24$. As $|P|=2$, we reach a contradiction.

CASE 2. $|G: F(G)|=3$.

In this case, by Lemma 3.2 , we get that $F(G)$ is abelian. It follows by Lemma 4.1 and Lemma 4.8 that $\left|F(G): G^{\prime}\right|=2$. Observe that $G \backslash F(G)=$ $x_{1} G^{\prime}+x_{2} G^{\prime}+x_{3} G^{\prime}+x_{4} G^{\prime}, x_{i} \in G \backslash F(G)$, where $x_{1}$ is a 3 -element of $G$. We easily see that $\left|C_{G}\left(x_{i}\right)\right|=6$. As $x_{1}$ is a 3-element of $G,\left|C_{G}(x)\right|=6$ implies that $|G|_{3}=3$. Set $P \in \operatorname{Syl}_{3}(G)$. Then $|P|=3$. Note that $F(G)$ is abelian. By Fitting lemma, we get $F(G)=C_{F(G)}(P) \times[F(G), P]$. Obviously, $C_{F(G)}(P)=Z(G)$ and $|Z(G)|=2$ since $\left|C_{G}(g)\right|=6$ for every $g \in G \backslash F(G)$. So, $G=B \times Z(G)$, where $B=[F(G), P] P$. Observe that $B$ is a Frobenius group with kernel $B^{\prime}=G^{\prime}=[F(G), P]$ and complement of order 3. Hence, $G$ satisfies (2) of the theorem.

CASE 3. $|G: F(G)|=4$.

In this case, applying again Lemma 4.1, we get that $G^{\prime}=F(G)$. Clearly, $G=K P$, where $K \leq G^{\prime}$ is a normal 2-complement of $G$ and $P \in \operatorname{Syl}_{2}(G)$. If $P$ is non-abelian, then by Lemma 4.4, it is impossible (note that if $G$ has the structure described in Lemma 4.4(2), then $K=F(G)<G^{\prime}$, but we have $G^{\prime}=F(G)$ ). In the following, we suppose that $P$ is abelian and so $|P|=4$. Then it follows from the hypothesis and Lemma 3.3 that $G^{\prime}$ is abelian.

We may assume that $G \backslash G^{\prime}=x G^{\prime}+y G^{\prime}+z G^{\prime}, x, y, z \in G \backslash G^{\prime}$. Since $k_{G}\left(G \backslash G^{\prime}\right)=4$, we may assume that both $x G^{\prime} \cup y G^{\prime}$ and $z G^{\prime}$ are a union of two conjugacy classes of $G$, respectively. Set $z G^{\prime}=z^{G}+h^{G}$. we have

and

$$
|G \backslash F(G)|=\frac{|G|}{\left|C_{G}(x)\right|}+\frac{|G|}{\left|C_{G}(y)\right|}+\frac{|G|}{\left|C_{G}(z)\right|}+\frac{|G|}{\left|C_{G}(h)\right|},
$$

It follows that

$$
\left|C_{G}(x)\right|=\left|C_{G}(y)\right|=4 .
$$

$$
\frac{3}{4}=\frac{1}{4}+\frac{1}{4}+\frac{1}{\left|C_{G}(z)\right|}+\frac{1}{\left|C_{G}(h)\right|} .
$$


Hence $\left|C_{G}(z)\right|=6$ and $\left|C_{G}(h)\right|=12$ (note that $|P|=4$ ). Thus $G$ satisfies (3) of the theorem.

CASE 4. $|G: F(G)|=5$.

Clearly, $G^{\prime}=F(G)$. It follows by Lemma 4.8 that $G$ is a Frobenius group with kernel $G^{\prime}$ and complement of order 5 . Then by Lemma 3.5, we have that $G^{\prime}$ is abelian, and so $G$ satisfies (4) of the theorem. The proof is completed.

\section{Proof of Theorem 1.2}

For a finite solvable group $G$, we define characteristic subgroup $F_{i}(G)$ by letting $F_{1}(G)=F(G)$, the unique largest nilpotent normal subgroup of $G$, and $F_{i+1}(G) / F_{i}(G)=F\left(G / F_{i}(G)\right)$. The nilpotent length (or the Fitting height) of a group $G$, denoted by $\operatorname{nl}(G)$, is the smallest number $l$ for which $F_{l}(G)=G$.

The following two results, which can be found in [14], will turn out to be useful in proof of Theorem 1.2.

Lemma 5.1. Let $G$ be a solvable group. Then $\operatorname{nl}(G) \leq(2 m(G)+5) / 3$, where $m(G)$ denotes the maximal number of conjugacy classes of $G$ on which some $\chi \in \operatorname{Irr}(G)$ vanishes.

Lemma 5.2. Suppose that $\operatorname{nl}(G) \geq 2$. If $\left|F_{2}(G): F(G)\right|$ is not prime, then there exists $\chi \in \operatorname{Irr}(G)$ such that $\chi$ vanishes on at least two conjugacy classes of $G$ contained in $F_{2}(G)-F(G)$.

Now we are ready to prove Theorem 1.2.

Proof of Theorem 1.2. Since $G$ is solvable, the hypothesis together with Lemma 5.1 yield that $\operatorname{nl}(G) \leq 4$. If $\operatorname{nl}(G)=1$, then by Corollary 2.5, $G \cong \mathrm{D}_{8}$ or $\mathrm{Q}_{8}$. Thus $G$ is a $V 3 C$-group. Hence we may assume that $\operatorname{nl}(G)=2$, 3 or 4 .

CASE $1 . \operatorname{nl}(G)=2$.

In this case, if $G / F(G)$ is non-abelian, then by Corollary $2.5, G / F(G) \cong$ $\mathrm{D}_{8}$ or $\mathrm{Q}_{8}$. Set $N / F(G)=(G / F(G))^{\prime}$. We have that $|G: N|=4$ and that $G \backslash N \subseteq \operatorname{Van}(G)$. Then it follows by Lemma 4.1 that $N=G^{\prime}$, and thus $G=K P$, where $K<G^{\prime}$ is a normal 2-complement of $G$ and $P \in \operatorname{Syl}_{2}(G)$. Thus Lemma 4.4 implies that $G$ is a Frobenius group with complement $Q_{8}$ (note that $\operatorname{nl}(G)=2$ ). Thus $G$ satisfies (1) of the Theorem. Hence we assume that $G / F(G)$ is abelian.

Since $G / F(G)$ is abelian, it follows by Lemma 4.3 that $G \backslash F(G) \subseteq \operatorname{Van}(G)$, and so $k_{G}(G \backslash F(G)) \leq 4$. If $k_{G}(G \backslash F(G))=1$, then $G$ is a Frobenius group with kernel $G^{\prime}$ and complement of order 2, and thus $G$ is a $V 3 C$-group. Therefore, $k_{G}(G \backslash F(G))=2,3$ or 4 .

CAse 1.1. $k_{G}(G \backslash F(G))=2$. 
In the case, note that $G \backslash F(G) \subseteq \operatorname{Van}(G)$, then by the hypothesis and Proposition 4.6, we have to the following three cases.

CASE 1.1.1. $G$ is a Frobenius group with kernel $G^{\prime}$ and complement of order 3 .

In this case, by Theorem $3.4, G^{\prime}$ is abelian. Thus $G$ is a $V 3 C$-group.

CAse 1.1.2. $G=G^{\prime} P$, where $G^{\prime}<F(G)$ and $G^{\prime}$ is a normal 2complement of $G$, and $P \in \operatorname{Syl}_{2}(G),|P|=4$.

In this case, observe that $F(G)=G^{\prime} \times O_{2}(G)$ and $O_{2}(G)=Z(G)$. Observe that $k_{G / Z(G)}(G / Z(G) \backslash F(G) / Z(G))=1$, then $G / Z(G)$ is a Frobenius group with Frobenius kernel $(G / Z(G))^{\prime} \cong G^{\prime}$ and complement of order 2. Then $G$ is of type (5) of Theorem 1.4, so a $V 3 C$-group.

CASE 1.1.3. $G=K P$, where $K<G^{\prime}$ is a normal abelian 2-complement of $G, P \in \operatorname{Syl}_{2}(G), P \cong \mathrm{D}_{8}$ or $\mathrm{Q}_{8},\left|G: G^{\prime}\right|=4$, and $G^{\prime}$ is abelian, $G \backslash G^{\prime}$ is a union $x^{G} \cup y^{G} \cup z^{G} \cup h^{G}$ of four conjugacy classes satisfying $\left|C_{G}(x)\right|=4$, $\left|C_{G}(y)\right|=4,\left|C_{G}(z)\right|=6$ and $\left|C_{G}(h)\right|=12$.

As $k_{G}(G \backslash F(G))=2$, we get that $|G: F(G)|=2$ and $\left|F(G): G^{\prime}\right|=2$. Clearly, $F(G)=K \times O_{2}(G)$ and so $F(G)$ is abelian. Note that 12||$F(G) \mid$ and hence 12||$C_{G}(g) \mid$ for any $g \in F(G)$ yielding a contradiction.

CASE 1.2. $k_{G}(G \backslash F(G))=3$. Thus $G \backslash F(G)$ is a union $x^{G} \cup y^{G} \cup z^{G}$ of three conjugacy classes of $G$.

Assume that $G$ is of type (8) of Proposition 4.7. Recall that $\operatorname{Van}(\operatorname{GL}(2,3))$ contains 6 conjugacy classes of $\operatorname{GL}(2,3)$ (see [6, P.161]), hence we may assume that $G / O_{2^{\prime}}(G)$ is isomorphic to a non-abelian group of order 16. It follows by Corollary 2.5 that the case also does not occur.

Assume that $G$ is of type (5) of Proposition 4.7. Then $|G: F(G)|=4$ and $G$ is a Frobenius group with complement $\mathrm{Q}_{8}$, which is impossible (as $F(G)$ is the Frobenuis kernel). From the hypothesis and Proposition 4.7, we only need to consider the following three cases:

CAsE 1.2.1. $G$ is a Frobenius group with abelian kernel $G^{\prime}$ and cyclic complement of order 4 .

In this case, $G$ is of type (4) of Theorem 1.4 , so a $V 3 C$-group.

CAse 1.2.2. $|G: F(G)|=2,\left|C_{G}(x)\right|=\left|C_{G}(y)\right|=\left|C_{G}(z)\right|=6$, and $F(G)$ is of odd order and has an abelian 3-complement.

In this case, applying the hypothesis and Lemma 3.1, we get that $F(G)$ is abelian. If $G^{\prime}=F(G)$, then Lemma 4.8 yields that $G$ is a Frobenius group with kernel $G^{\prime}$ and cyclic complement of order 2, and thus $G$ is of type (1) of Theorem 1.4, so a $V 3 C$-group. Hence $G^{\prime}<F(G)$. Furthermore, applying again by Lemma 4.1 , we have that $\left|F(G): G^{\prime}\right|=3$.

Recall that $G \backslash F(G)=x G^{\prime}+y G^{\prime}+z G^{\prime}$, where $x, y, z \in G \backslash F(G)$. Then by the second orthogonality relation we have

$$
6=\left|C_{G}(g)\right|=\left|G / G^{\prime}\right|+\sum\left\{|\chi(g)|^{2} \mid \chi \in \operatorname{Irr}_{1}(G)\right\},
$$

for all $g \in G \backslash F(G)$. Hence $\chi(g)=0$ for all $g \in G \backslash F(G)$ and all $\chi \in \operatorname{Irr}_{1}(G)$. 
Set $P \in \operatorname{Syl}_{2}(G)$. Note that $|G: F(G)|=2$ and that $F(G)$ is of odd order. Let $P=\langle t\rangle$, where $t$ is an involution. By Fitting Lemma, we have $F(G)=C_{F(G)}(P) \times[F(G), P]$. Obviously, $C_{F(G)}(t)=C_{F(G)}(P)=Z(G)$. Since $\left|C_{G}(g)\right|=6$ for every $g \in G \backslash F(G)$, we conclude that $|Z(G)|=3$. So, $G=B \times Z(G)$, where $B=[F(G), P] P$. Observe that $B$ is a Frobenius group with kernel $B^{\prime}=G^{\prime}=[F(G), P] G^{\prime}$ and complement of order 2. Then $G$ is of type (7) of Theorem 1.4 , so a $V 3 C$-group.

CAse 1.2.3. $|G: F(G)|=2,\left|C_{G}(x)\right|=4,\left|C_{G}(y)\right|=6$ and $\left|C_{G}(z)\right|=12$.

In this case, it follows by Lemma 3.1 that $F(G)$ is abelian. Recall now that $G=K P$, where $K \leq G^{\prime}$ is a normal 2-complement of $G$ and $P \in \operatorname{Syl}_{2}(G)$. It follows by Lemma 4.4 that $P$ is abelian (note that $|G: F(G)|=2$ ), and so $K=G^{\prime}$ and $|P|=4$.

Notice that $|G: F(G)|=2$ and $F(G)$ is abelian. It easily follows that $F(G)=G^{\prime} \times Z(G)$ and that $Z(G)$ is of order 2 . Then we have that $[G / Z(G)$ : $\left.(G / Z(G))^{\prime}\right]=[G / Z(G): F(G) / Z(G)]=2$, and thus it follows by Lemma 4.8 that $G$ is of type (5) of Theorem 1.4 , so a $V 3 C$-group.

CASE 1.3. $k_{G}(G \backslash F(G))=4$.

Note that $G / F(G)$ is abelian and $G \backslash F(G)=\operatorname{Van}(G)$. Then applying Theorem 4.9, $G$ satisfies (2), (3), (4) or (5) of Theorem 1.2.

Case 2. $\operatorname{nl}(G)=3$.

Form the proof of the case when $\operatorname{nl}(G)=2$, we see that if $\operatorname{nl}(G)=2$ then $G \backslash F(G) \subseteq \operatorname{Van}(G)$ and $F(G)$ is abelian. So, if $\operatorname{nl}(G)=3$, then $G / F(G) \backslash F_{2}(G) / F(G) \subseteq \operatorname{Van}(G / F(G))$ and $F_{2}(G) / F(G)$ is abelian. It follows by Lemma 4.3 that $F_{2}(G) \backslash F(G) \subseteq \operatorname{Van}(G)$, and thus $G \backslash F(G) \subseteq$ $\operatorname{Van}(G)$ (note that $G \backslash F_{2}(G) \subseteq \operatorname{Van}(G)$ ). Hence the hypothesis yields that $k_{G / F(G)}(G / F(G)) \leq 5$. Since $\operatorname{nl}(G / F(G))=2$, it follows by [18] that $G / F(G)$ is isomorphic to one of the following groups: $\mathrm{S}_{3}, \mathrm{D}_{10}, \mathrm{~A}_{4}, \mathrm{D}_{14},\left(\mathrm{C}_{4}, \mathrm{C}_{5}\right)$ or $\left(\mathrm{C}_{3}, \mathrm{C}_{7}\right)$.

From the proof of paragraph above, we have that both $G \backslash F_{2}(G)$ and $F_{2}(G) \backslash F(G)$ are contained in $\operatorname{Van}(G)$. Since $F_{2}(G) \backslash F(G)$ contains at least one conjugacy class of $G$, it follows by the hypothesis that $k_{G}\left(G \backslash F_{2}(G)\right)=2$ or 3 (if $k_{G}\left(G \backslash F_{2}(G)\right)=1$, then $G$ is a Frobenius group with kernel $G^{\prime}$ and complement of order 2 , and thus $G$ is a $V 3 C$-group.).

CAsE 2.1. $k_{G}\left(G \backslash F_{2}(G)\right)=2$.

In this case, by Proposition 4.6, we have to discuss the following two cases:

CASE 2.1.1. $\left|F_{2}(G): G^{\prime}\right|=2,\left|G: F_{2}(G)\right|=2, G=G^{\prime} P$, where $G^{\prime}$ is a normal 2-complement of $G$, and $P \in \operatorname{Syl}_{2}(G),|P|=4$.

Note that $\left[G: F_{2}(G)\right]=2$ and $k_{G}\left(F_{2}(G) \backslash F(G)\right) \leq 2$, we have that $G / F(G) \cong \mathrm{S}_{3}$ or $\mathrm{D}_{10}$, and thus $\left|G / F(G):(G / F(G))^{\prime}\right|=2$. If $O_{2}(G)=1$, then $F(G) \leq G^{\prime}$ and so $\left|G / F(G):(G / F(G))^{\prime}\right|=\left|G: G^{\prime}\right|=4$, a contradiction. Hence $\left|O_{2}(G)\right|=2$ and so $O_{2}(G)=Z(G)$. Clearly,

$$
k_{G / Z(G)}\left(G / Z(G) \backslash F_{2}(G) / Z(G)\right)=1
$$


so $G / Z(G)$ is a Frobenius group with kernel $(G / Z(G))^{\prime} \cong G^{\prime}$ and complement $P / Z(G)$ of order 2. Then $G^{\prime}$ is abelian and thus $G^{\prime} \leq F(G)$, a contradiction (note that $\operatorname{nl}(G)=3$ ).

CASE 2.1.2. $G^{\prime}=F_{2}(G),\left|G: G^{\prime}\right|=2$ and $G / O_{2^{\prime}}(G) \cong \mathrm{S}_{4}$.

Write $\bar{G}=G / O_{2^{\prime}}(G)$. Then $F_{2}(G) / O_{2^{\prime}}(G)=F_{2}(\bar{G})$. Observe that $G / F(G) \cong \mathrm{S}_{3}$ or $\mathrm{D}_{10}$; thus $\left|O_{2}(G)\right|=4$ and so $\overline{F(G)}=F(\bar{G})$. Hence we easily see that $G / F(G) \cong \mathrm{S}_{3}$ and that $F(G)=O_{2}(G) \times O_{2^{\prime}}(G)$.

Assume that $F_{2}(G) \backslash F(G)=x^{G}+y^{G}$, where $x$ is a 3 -element of $G$. Observe that $\left|C_{G}(x)\right|=\left|C_{G}(y)\right|=6$ and so $x$ is an element of order 3 . we easily see that $x$ commutes with an involution $t$ in $F(G)$, we obtain a contradiction (note that $\overline{F_{2}(G)}$ is a Frobenius group with kernel $\overline{F(G)}$ ). The contradiction shows that $k_{G}\left(F_{2}(G) \backslash F(G)\right)=1$ and so $k_{G}(G \backslash F(G))=3$. Recall that $G / F(G) \cong \mathrm{S}_{3}$; then by Proposition $4.7, G \cong \mathrm{S}_{4}$ and thus $G$ is of type (6) of Theorem 1.4 , so a $V 3 C$-groups.

CAse 2.2. $k_{G}\left(G \backslash F_{2}(G)\right)=3$.

Recall that $\operatorname{nl}(G)=3$. We then in a position to apply Proposition 4.7 to $G$, with $F_{2}(G)$ playing the role of $N$, obtaining that $G$ is of types (6), (7) or (8) of Proposition 4.7. In particular, $\left[G: F_{2}(G)\right]=2$. On the other hand, it easily see that $k_{G}\left(F_{2}(G) \backslash F(G)\right)=1$, and so $F_{2}(G) / F(G)$ contains exactly two conjugacy classes of $G / F(G)$. Hence $G / F(G) \cong \mathrm{S}_{3}$. Set $F_{2}(G) \backslash F(G)=g^{G}$ such that $g$ is a 3 -element. Observe that $\left|C_{G}(g)\right|=3$ and thus $|G|_{3}=3$. Recall that $k_{G}\left(G \backslash F_{2}(G)\right)=3$ and $G / F(G) \cong \mathrm{S}_{3}$; thus $\left|G: F_{2}(G)\right|=2$ and $G$ satisfies (6) or (7) of Proposition 4.7. Then $G$ contains an element $x$ with $\left|C_{G}(x)\right|=6$, which shows that there exists an element $y$ in $F_{2}(G) \backslash F(G)$ such that $C_{G}(y)$ contains an involution, a contradiction.

CASE 3. $\operatorname{nl}(G)=4$.

Then $\operatorname{nl}(G / F(G))=3$ and thus $G / F(G) \cong \mathrm{S}_{4}$. Consequently,

$$
G / F(G) \backslash F_{2}(G) / F(G) \subseteq \operatorname{Van}(G / F(G)) .
$$

It follows that $G \backslash F_{2}(G) \subseteq \operatorname{Van}(G)$. Note that $F_{2}(G) / F(G)$ is an elementary abelian group of order 4, then Lemma 4.3 together with Lemma 5.2 yield that $F_{2}(G) \backslash F(G) \subseteq \operatorname{Van}(G)$ and that $k_{G}\left(F_{2}(G) \backslash F(G)\right) \geq 2$, thus we reach a contradiction (recall that $k_{G}\left(G \backslash F_{2}(G)\right) \geq 3$ ). The contradiction completes the proof.

Lemma 5.3 ([1, Theorem 2.3]). There is some vanishing sum $v_{1}+v_{2}+$ $\ldots+v_{n}=0$ of $n$ mth roots of unity if and only if $n$ is a linear combination, with non-negative integer coefficients, of the prime divisors of $m$.

Corollary 5.4. Suppose that $\chi(1)=2$ or 4 for all $\chi \in \operatorname{Irr}_{1}(G)$. Then $\operatorname{Van}(G)$ cannot contain elements of odd orders.

Let $\chi$ be an irreducible character of $G$. Note that if $x \in v(\chi), z \in Z(G)$, then $x z \in v(\chi)$. Indeed, if $D$ is a representation of $G$ with character $\chi$, then 
$D(x z)=D(x) D(z)=(\lambda(z) I) D(x)$, where $\lambda$ is a linear character of $Z(G)$ and $I$ is the identity matrix with degree $\chi(1)$. So $\chi(x z)=\operatorname{tr}(D(x z))=\lambda(z) \chi(x)=$ 0 .

Remark 5.5. We show that $G$ is one of types except for (1) and (5) in Theorem 1.2, then $G$ is a $V 4 C$-group (but not a $V 3 C$-group). Suppose that $G$ is of type (2) of Theorem 1.2. Then by Corollary $5.4, F(G)$ does not contain vanishing elements of $G$ (note that $F(G)=G^{\prime} \times Z(G)$ ). Note that $[G: F(G)]=2$, thus by Lemma $4.3, G \backslash F(G)=\operatorname{Van}(G)$. Set $G \backslash F(G)=$ $n_{1}^{G}+\cdots+n_{s}^{G}$. Then, we get

It follows that

$$
|G \backslash F(G)|=\frac{|G|}{\left|C_{G}\left(n_{1}\right)\right|}+\cdots+\frac{|G|}{\left|C_{G}\left(n_{s}\right)\right|},
$$

$$
\frac{1}{2}=\frac{1}{\left|C_{G}\left(n_{1}\right)\right|}+\cdots+\frac{1}{\left|C_{G}\left(n_{s}\right)\right|} .
$$

Since $G / Z(G)$ is a Frobenius group with kernel $(G / Z(G))^{\prime} \cong G^{\prime}$ and complement $P / Z(G)$ of order $2, n_{i}$ acts fixed point freely on $G^{\prime}$, and so $\left|C_{G}\left(n_{i}\right)\right|=8$ (note that $P$ is abelian). Hence $s=4$. If $G$ satisfies (4) of Theorem 1.2, then by Corollary 5.4, $G$ is a $V 4 C$-group (but not a $V 3 C$ group). Clearly, the same is true if $G$ is of type (3) in Theorem 1.2.

It is worth mentioning that there exists Frobenius groups $G$ with complement of order 5 and abelian kernel $N$, such that $N \cap \operatorname{Van}(G)$ is nonempty (see [1, Example 2]), so $G$ is not a $V 4 C$-group.

In the following, we give three groups $G_{1}, G_{2}$ and $G_{3}$ satisfying (2), (3) and (4) in Theorem 1.2, respectively (see [18]).

$$
\begin{gathered}
G_{1}=[C(3)], \quad C(8)=\left\langle a, b \mid a^{8}=b^{3}=1, a^{-1} b a=b^{-1}\right\rangle, \\
G_{2}=A_{4} \times C(2), \quad G_{3}=[C(15)], \\
C(4)=\left\langle a, b, c \mid a^{5}=b^{3}=c^{4}=1, a b=b a, c^{-1} a c=a^{2}, c^{-1} b c=b^{-1}\right\rangle .
\end{gathered}
$$

However, if $G$ satisfies (1) of Theorem 1.2, that is, $G$ is a Frobenius group with kernel $M$ and complement $\mathrm{Q}_{8}$, then we do not know whether $M \cap \operatorname{Van}(G)$ is empty or not.

\section{Non-SOlvable $V 4 C$-GROUPS}

In this section, we study the non-solvable $V 4 C$-groups.

Let $p$ be a prime number. Recall that a character $\chi \in \operatorname{Irr}(G)$ is said to be of $p$-defect zero if $p$ does not divide $|G| / \chi(1)$. By a fundamental result of Brauer (see [8, Theorem 8.17]), if $\chi \in \operatorname{Irr}(G)$ is of $p$-defect zero then, for every element $g \in G$ such that $p$ divides $o(g)$, we have $\chi(g)=0$.

The following Lemma comes from [3, Proposition 2.1].

LEMma 6.1. Let $G$ be a non-abelian simple group and $p$ a prime number. If $G$ is of Lie type, or if $p \geq 5$, then there exists $\chi \in \operatorname{Irr}(G)$ of p-defect zero. 
Lemma 6.2. Let $G$ be a non-abelian simple group. If $G$ is a $V 4 C$-group, then $G$ is isomorphic to $A_{5}$.

Proof. Let $G$ be a simple $V 4 C$-group. Let $G \cong \mathrm{A}_{n}$ for some $n \geq$ 14. Then $\{5,10,7,14,11\} \subseteq \pi_{e}(G)$ and thus by Lemma 6.1 , we obtain a contradiction. Hence the hypothesis implies that $G \cong \mathrm{A}_{n}$ for some $n \leq 13$, and so $G \cong \mathrm{A}_{5}$ from $[2]$.

By [2], $G$ cannot be a sporadic simple groups. By the classification theorem of the finite simple groups we can now suppose that $G$ is a simple group of Lie type. Then, by Lemma 6.1 , for each prime factor $p$ of $|G|$ there exists some $\chi \in \operatorname{Irr}_{1}(G)$ such that $\chi$ is of $p$-defect zero. Hence any nonidentity element of $G$ is contained in $\operatorname{Van}(G)$. It follows by the hypothesis that $G$ consists of five conjugacy classes, and then, by [18], $G$ is isomorphic to $\mathrm{A}_{5}$.

Lemma 6.3. Let $G$ be non-solvable group. If $G$ is a $V 4 C$-group, then $G$ has the unique non-abelian composite factor.

Proof. By induction, we may assume that $\operatorname{Sol}(G)$, the maximal solvable normal subgroup of $G$, is trivial. Let $N$ be a (non-solvable) minimal normal subgroup of $G$. If $N$ is not a non-abelian simple group, then $N=N_{1} \times \ldots \times N_{s}$ is a direct product of isomorphic simple groups $N_{i}$, where $s \geq 2$. If $p \geq 5$, then there exists $\theta_{i} \in \operatorname{Irr}\left(N_{i}\right)$ of $p$-defect zero (see Lemma 6.1), and set

$$
\theta=\theta_{1} \times \ldots \times \theta_{s} .
$$

Let $\chi_{0}$ be an irreducible constituent of $\theta^{G}$, let $x_{1} \in N_{1}$ be of a prime order $p$, $x_{2} \in N_{2}$ be of a prime order $q$. Notice that

$$
x_{1}^{G} \subseteq N_{1} \cup \ldots \cup N_{s} .
$$

So $x_{1} x_{2}$ is not conjugate to $x_{1}$. Clearly, $\theta^{g}$ is of $p$-defect zero for any $g \in G$, then we have

This implies that

$$
\theta^{g}\left(x_{1}\right)=\theta^{g}\left(x_{1} x_{2}\right)=0
$$

$$
\chi_{0}\left(x_{1}\right)=\chi_{0}\left(x_{1} x_{2}\right)=0 .
$$

Then the hypothesis yields that $N_{1}$ has only one prime divisor $p$ greater than 3 , that $N_{1}$ is a simple $\mathrm{K}_{3}$-group (a simple group $G$ is called a simple $\mathrm{K}_{3^{-}}$ group if the number of prime factors of $|G|$ is 3 ), and also that $N_{1}$ has no irreducible character of 2-defect zero or 3-defect zero. By [2] and [5], we obtain a contradiction. Hence $N$ is a simple group.

Suppose that $G / N$ is non-solvable. Note that out $(N)$ is solvable by the the classification of the finite simple groups, it follows that $C_{G}(N)$ is nonsolvable and hence contains a non-solvable minimal normal subgroup $M$ of $G$ as $\operatorname{Sol}\left(C_{G}(N)\right)=1$. Set $T=M \times N$. Let $\psi \in \operatorname{Irr}(M)$ be $q$-defect zero, and let $\theta \in \operatorname{Irr}(N)$ be a $p$-defect zero, where $q, p$ are prime divisors of $|M|$ and $|N|$, respectively. Let $x_{1}, x_{2} \in M$ be of order $q, s$, respectively, where $s \neq p$ and 
$s \neq q$. Let $y_{1}, y_{2} \in N$ be of order $p, r$, respectively, where $r \neq p$ and $r \neq q$. Then for any irreducible constituent $\chi$ of $(\psi \times \theta)^{G}$, we see that

$$
\chi\left(x_{1}\right)=\chi\left(x_{1} y_{1}\right)=\chi\left(x_{1} y_{2}\right)=\chi\left(y_{1}\right)=\chi\left(y_{1} x_{2}\right)=0 .
$$

The contradiction completes the proof.

Lemma 6.4. Let $G$ be non-solvable group. If $G$ is a $V 4 C$-group, then $G$ is perfect (i.e. $G=G^{\prime}$ ).

Proof. Otherwise, we may assume that $G^{\prime}<G$. By Lemma 6.3, there exist two normal subgroups $N$ and $M$ of $G$ such that $N<M \leq G^{\prime}$ and $M / N$ is a non-abelian simple group. From the argument of the proof of Lemma 6.3, we get that $(M / N) \cap \operatorname{Van}(G / N)$ is non-empty, and thus $G^{\prime} \cap \operatorname{Van}(G)$ is also non-empty.

Suppose that there exists $\chi \in \operatorname{Irr}(G)$ such that $\chi_{G^{\prime}}$ is not irreducible. It follows by [8, Theorem 6.22] that $G$ has a proper subgroup $H$ such that $G^{\prime} \leq H<G$ and $G \backslash H \subseteq v(\chi)$. Since $G^{\prime} \cap \operatorname{Van}(G)$ is also non-empty, it follows by the hypothesis that $k_{G}(G \backslash H) \leq 3$. If $k_{G}(G \backslash H) \leq 2$, then $G$ is solvable (see [15, Theorem 2.2]), a contradiction. Hence we may assume that $k_{G}(G \backslash H)=3$. Note that $G$ is non-solvable; it follows by [15, Theorem 3.5] that $G$ has a normal subgroup $E$ such that $G / E \cong \mathrm{S}_{5}$ or $\mathrm{M}_{10}$, then we obtain a contradiction from [2] and the proof is complete.

Lemma 6.5 ([12, Theorem]). Suppose that a group $G$ contains a subgroup $X$ of order 3 such that $C_{G}(X)=X$. If,for every $g \in G$, the subgroup $\left\langle X, X^{g}\right\rangle$ is finite, then one of the following holds:

(1) $G=N N_{G}(X)$ for a periodic nilpotent subgroup $N$ of nilpotent class 2, and $N X$ is a Frobenius group with kernel $N$ and complement $X$.

(2) $G=N A$, where $A$ is isomorphic to $A_{5} \cong S L_{2}(4)$ and $N$ is a normal elementary Abelian 2-subgroup, here, $N$ is a direct product of order 16 subgroups normal in $G$ and isomorphic to the natural $S L_{2}(4)$-module of dimension 2 over a field of order 4.

(3) $G$ is isomorphic to $L_{2}(7)$.

Next we are ready to prove Theorem 1.3.

Proof of Theorem 1.3. Clearly our hypothesis is inherited by any factor group. By Lemma 6.2, it suffices to show that $G$ is a non-abelian simple group. Assume that $G$ is a minimal counter-example.

First, we show that $G$ has a minimal normal subgroup $N$ such that $G / N$ is non-solvable. Otherwise, we may assume that $G$ has the unique minimal normal subgroup $N$ such that $G / N$ is solvable. Recall that $(G / N)^{\prime}=G^{\prime} N / N$, thus it follows by Lemma 6.4 that $(G / N)^{\prime}=G / N$, which yields that $N=G$, a contradiction (note that $G$ is a minimal counter-example). Hence $G / N$ is non-solvable. Now it follows by induction that $G / N$ is a non-abelian simple 
group. Applying Lemma 6.2, we conclude that $G / N \cong \mathrm{A}_{5}$. Then $G / N$ has exactly one conjugacy class of elements of order 3 . Choose a 3-element $a$ of $G$ such that $(a N)^{G / N}$ is the class of elements of order 3 in $G / N$. Set $A=(a N)^{G / N}$.

Recall that $k_{G / N}(\operatorname{Van}(G / N))=4$; thus $k_{G}(A)=1$. Then each $\chi \in$ $\operatorname{Irr}(G \mid N)$ vanishes on $A$. By the second orthogonality relation we have

$$
\left|C_{G}(a)\right|=\left|C_{G / N}(a N)\right|=3 .
$$

Hence $G$ has an element $a$ with $C_{G}(a)$ of order 3 . Hence $G$ satisfies the hypothesis of Lemma 6.5 .

If $G$ is the group in Lemma $6.5(1)$, then $G$ is solvable, which is a contradiction.

Suppose that $G$ has the structure described in Lemma 6.5(3). Then we obtain a contradiction from [2]. Hence $G$ is the group in Lemma 6.5(2). But $G$ is not a $V 4 C$-group (see [18, p. 310]), which is the final contradiction.

\section{ACKNOWLEDGEMENTS.}

The authors are grateful to the referee for pointing out some inaccuracies in an earlier version of the paper as well as for helpful comments that greatly improved the exposition of the paper. In particular, the authors are grateful to the referee who improved Lemma 6.4. This simplified the proof of Theorem 1.3. This paper is supported by the NNSF of China (11201401, 11301532, 11371307), the Sichuan Provincial Education Department Foundation of China (10ZB098) and the NSF of Sichuan University of Science and Engineering (2009XJKRL011).

\section{REFERENCES}

[1] D. Bubboloni, S. Dolfi and P. Spiga, Finite groups whose irreducible characters vanish only on p-elements, J. Pure Appl. Algebra 213 (2009), 370-376.

[2] J. H. Conway, R. T. Curtis, S. P. Norton, R. A. Parker and R. A. Wilson, Atlas of finite groups, Oxford University Press, Eynsham, 1985.

[3] S. Dolfi, E. Pacifici, L. Sanus and P. Spiga, On the orders of zeros of irreducible characters, J. Algebra 321 (2009), 345-352.

[4] S. Dolfi, E. Pacifici, L. Sanus and P. Spiga, On the vanishing prime graph of solvable groups, J. Group Theory 13 (2010), 189-206.

[5] M. Herzog, On finite simple groups of order divisible by three primes only, J. Algebra 10 (1968), 383-388.

[6] B. Huppert, Character theory of finite groups, de Gruyter, Berlin, 1998.

[7] B. Huppert, Endliche gruppen, Vol. 1, Springer-Verlag, Berlin-New York, 1967.

[8] I. M. Isaacs, Character theory of finite groups, Academic Prees, New York-London 1976.

[9] I. M. Isaacs, G. Navarro and T. R. Wolf, Finite group elements where no irreducible character vanishes, J. Algebra 222 (1999), 413-423.

[10] G. James and M. Liebeck, Representations and character of groups, Cambridge University Press, Cambridge, 1993. 
[11] O. Manz and T. R. Wolf, Representations of solvable groups, Cambridge University Press, Cambridge, 1993.

[12] V. D. Mazurov, On groups that contain a self-centralizing subgroup of order 3 , Algebra and Logic 42 (2003), 29-36.

[13] A. Moretó and J. Sangroniz, On the number of conjugacy classes of zeros of characters, Israel J. Math. 142 (2004), 163-187.

[14] G. Qian, Bounding the fitting height of a solvable group by the number of zeros in a character table, Proc. Amer. Math. Soc., 142 (2002), 3171-3176.

[15] G. Qian, W. Shi and X. You, Conjugacy classes outside a normal subgroup, Comm. Algebra 32 (2004), 4809-4820.

[16] Y. C. Ren, X. H. Liu and J. S. Zhang, Notes on the restriction and the zeros of an irreducible character of a finite group, Sichuan Daxue Xuebao 44 (2007), 1183-1188.

[17] C. Shao, S. Humphries, X. You and J. Zhang, A note on conjugacy classes outside a normal subgroup, Comm. Algebra 37 (2009), 3306-3308.

[18] A. Vera Lopez and J. Vera Lopez, Classification of finite groups according to the number of conjugacy classes, Israel J. Math. 51 (1985), 305-338.

[19] W. J. Wong, Finite groups with a self-centralizing subgroup of order 4, J. Austral. Math. Soc. 7 (1967), 570-576.

[20] J. S. Zhang, W. J. Shi and Z. C. Shen, Finite groups in which every irreducible character vanishes on at most three conjugacy classes, J. Group Theory 13 (2010), 799-819.

J. Zhang

School of Science

Sichuan University of Science and Engineering

643000 Zigong

P. R. China

E-mail: zjscdut@163.com

Z. Shen

College of Science

China Agricultural University

100083 Beijing

P. R. China

E-mail: zhencai688@sina.com

J. Shi

School of Mathematics and Information Science

Yantai University

264005 Yantai

P. R. China

E-mail: shijt@math.pku.edu.cn

Received: 9.3.2013.

Revised: 30.8.2013. \& 6.9.2013. 